\title{
Comparação entre o Método da Gota Pendente e 0 Método da Gota Girante para Medida da Tensão Interfacial entre Polímeros
}

\author{
Nicole R. Demarquette e Musa R. Kamal
}

\begin{abstract}
Resumo: Dois instrumentos, um deles baseado no princípio da gota pendente e o outro baseado no método da gota girante, para medir a tensão interfacial entre polímeros, são apresentados e comparados aqui. Com ambos instrumentos foi possível visualizar a gota de polímero “on line”. Os instrumentos mostraram-se complementares quanto às suas áreas de aplicação. O método da gota pendente deve ser usado quando as quantidades de polímero são limitadas ou quando o polímero mais denso é opaco. O método da gota girante deve ser usado quando a degradação térmica pode ser um problema ou quando o polímero menos denso é opaco.
\end{abstract}

Palavras-chave: Tensão interfacial, blendas poliméricas

\section{Introdução}

Uma das áreas de materiais que mais tem despertado a atenção no mundo é a que inclui as chamadas "blendas poliméricas" (polymer blends). Blendas poliméricas são originadas quando dois ou mais polímeros ou copolímeros são mecanicamente misturados sob fusão. Misturas de polímeros são cada vez mais utilizadas nas indústrias de embalagens, especialmente substituindo embalagens que utilizam camadas múltiplas. Essas misturas combinam as propriedades dos diferentes componentes, resultando em materiais que podem ter grande resistência mecânica, baixa permeabilidade à água e ao oxigênio, opacidade e outras características que os tornam extremamente interessantes para numerosas aplicações. Outras vantagens desses materiais incluem: baixo preço, uma única operação de processo, possi- bilidade de reciclagem e baixo investimento de capital para fabricação.

Foi demonstrado que as características finais (propriedades mecânicas, barreiras) de uma mistura de polímeros dependem da sua morfologia, que por sua vez depende das dimensões da fase dispersa e da tensão interfacial entre os componentes da mistura. Portanto, um parâmetro muito importante na caracterização de blendas poliméricas é a tensão interfacial entre os constituintes. A tensão interfacial é o parâmetro chave no controle da compatibilidade entre os constituintes de uma mistura de polímeros ${ }^{1,2}$. A tensão interfacial é também o parâmetro mais acessível que descreve as condições termodinâmicas e estruturais das interfaces das misturas. Vários trabalhos têm sido conduzidos em todo o mundo para determinar com exatidão os efeitos das tensões interfaciais nas propriedades das misturas de polímeros ${ }^{1,3}$. Entretanto é

Nicole R. Demarquette, Escola Politécnica da Universidade de São Paulo - Departamento de Engenharia Metalúrgica e de Materiais, Av. Prof. Mello Moraes, 2463, Cidade Universitária, 05508-900, São Paulo, SP, Musa R. Kamal, McGill University, Chemical Engineering Department, 3480 University Street, H3A 2 A7 Montreal, Quebec, Canada 
muito difícil medir a tensão interfacial entre polímeros. Os polímeros são muito viscosos e como consequência os ensaios para a determinação da tensão interfacial são muito demorados e problemas de degradação podem acontecer.

Os primeiros estudos sobre tensão interfacial entre polímeros foram publicados somente há cerca de 30 anos atrás ${ }^{4,5}$. Desde então, vários métodos para medir a tensão interfacial foram desenvolvidos ${ }^{6}$. Entre os métodos disponíveis somente poucos podem ser usados para medir a tensão interfacial entre polímeros. Os métodos de determinação da tensão interfacial entre polímeros podem ser divididos em duas categorias; estáticos e dinâmicos. Em geral, os métodos estáticos são os mais usados. Esses métodos baseados na determinação do perfil de uma gota pendente, (pendant drop) (4,5-7-10 $^{\text {, séssil }}{ }^{11-13}$ ou gota girante (spinning drop) ${ }^{14-17}$. Os métodos dinâmicos como instabilidade de fibra fundida (molten thread $)^{18}$ ou retração de fibras imbutidas (imbedded fibers) $)^{19}$ são também usados para avaliar a tensão interfacial entre polímeros. Métodos dinâmicos baseados na dispersão de luz superficial ${ }^{20-21}$ foram sugeridos e estão sendo desenvolvidos. Grassmespacher e Meissner ${ }^{22}$ e Graebling ${ }^{23}$ desenvolveram uma teoria para inferir a tensão interfacial entre os componentes de uma blenda através de medidas das propriedades reológicas da blenda.

Há ainda métodos usados para medir a tensão interfacial entre líquidos, entre eles estão a técnica do capilar (capillary rise technique) ${ }^{24-26}$, das placas Wilhelmy ${ }^{27}$, do anel de Nouy ${ }^{28-30}$, do peso da gota $^{31}$, e da pressão máxima na bolha ${ }^{25}$. Entretanto, esses métodos não podem ser aplicados na medida da tensão interfacial entre polímeros devido à alta viscosidade desse materiais.

Este artigo apresenta dois instrumentos, um deles baseado no método da gota pendente e o outro no método da gota girante utilizados na medida da tensão interfacial entre polímeros. É apresentada uma comparação entre os dois métodos.

\section{Método da gota pendente}

\section{Princípio}

Dentre todos os possíveis métodos para se determinar a tensão interfacial entre polímeros o método da gota pendente é provavelmente o mais popular.
Consiste na determinação do perfil de uma gota pendente de um líquido suspenso em um outro líquido mais denso. O perfil da gota, quando está em equilíbrio mecânico, é determinado por um balanço entre a força de gravidade e as forças de superfície, e pode ser descrito por uma equação diferencial que não possui soluções analíticas ${ }^{32}$ (equações 1a,1b, 1c, 1d):

$$
2 \neq \mathrm{B} \frac{\mathrm{z}}{\mathrm{a}}=\frac{1}{\frac{\mathrm{R}_{1}}{\mathrm{a}}} \neq \frac{\sin \phi}{\frac{\mathrm{x}^{5}}{\mathrm{a}}}
$$

onde

$$
\mathrm{B}=\frac{\mathrm{a}^{2} \Delta \rho \mathrm{g}}{\gamma}
$$

e

$$
\begin{aligned}
& \mathrm{R}_{1}=\frac{\mathrm{ds}}{\mathrm{d} \phi} \frac{\left\{1+\left(\frac{\mathrm{dz}}{\mathrm{dx}}\right)^{2}\right\}^{\frac{3}{2}}}{\frac{\mathrm{d}^{2} \mathrm{z}}{\mathrm{d}^{2} \mathrm{x}}} \\
& \sin \phi=\frac{\frac{\mathrm{dz}}{\mathrm{dx}}}{\left\{1+\left(\frac{\mathrm{dz}}{\mathrm{dx}}\right)^{2}\right\}^{\frac{1}{2}}}
\end{aligned}
$$

$\Delta \rho$ é a diferença de densidade dos dois líquidos em contato, g é a aceleração gravitacional, $\gamma$ é a tensão interfacial, a é o raio de curvatura no ápex da gota, $\mathrm{x}, \mathrm{z}, \phi$ são as coordenadas definidas como na Figura 1, e $\mathrm{R}_{1}$ é o raio de curvatura no ponto $\mathrm{x}, \mathrm{y}$.

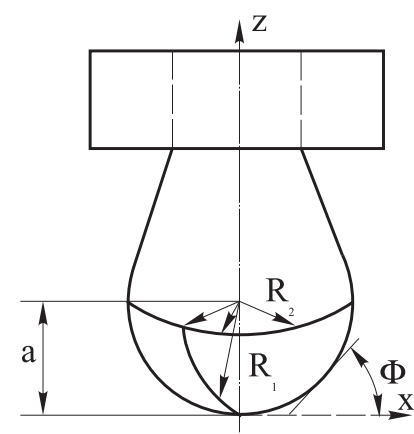

Figura 1. Geometria de uma gota pendente 
O método da gota pendente apresenta vantagens quando comparado com outros métodos: é um método absoluto, a interface não é perturbada durante o experimento e o arranjo experimental é simples.

\section{Descrição do instrumento usado neste trabalho}

O aparelho desenvolvido, baseado no método da gota pendente é composto de: i) uma câmara experimental aquecida onde a gota é formada; ii) de um sistema óptico para iluminar e capturar a imagem da gota; iii) de um sistema de aquisição de dados com um computador para inferir a tensão interfacial do perfil da gota. A Figura 2 apresenta um esquema do aparelho. Os diferentes componentes do aparelho são montados sobre uma mesa com amortecedores de vibração. É muito importante que os ensaios sejam realizados neste tipo de mesa para se evitar a separação da gota.

O sistema de iluminação consiste em uma lâmpada e fibra óptica. O comprimento de onda da luz pode ser modificado usando-se filtros quando a gota e a matriz têm o mesmo índice de refração para um dado comprimento de onda.

O sistema de visualização da gota é composto de um sistema óptico que aumenta a imagem da gota e a projeta em um equipamento de digitação de imagens. Uma câmara de vídeo é acoplada ao sistema óptico levando a imagem até a digitalizador residente no computador. O digitalizador tem uma resolução de 480x640 pixels.

A câmara experimental é composta de um vaso de secção quadrada de aço inoxidável, aquecido eletricamente, no qual um cilindro em cobre é inserido. Uma célula de vidro, do mesmo tipo utilizada em espectroscopia UV, é inserida no cilindro de cobre. Nessa célula é inserido o polímero de menor densidade. Duas janelas de vidro, em dois lados opostos da câmara experimental, permitem a iluminação e

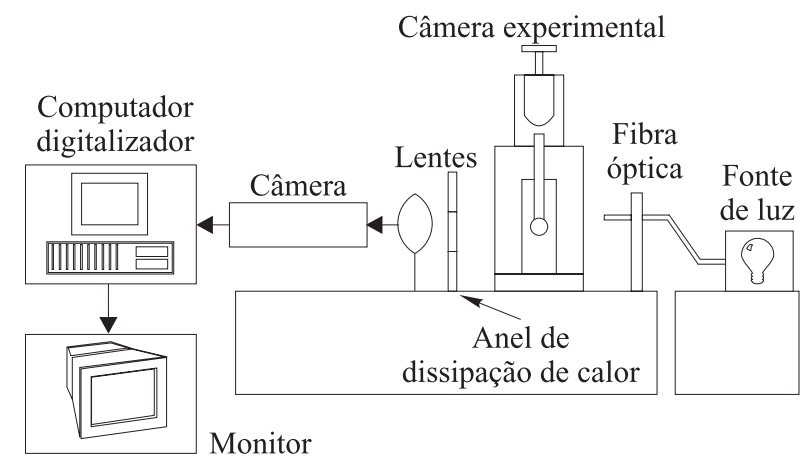

Figura 2. Esquema do instrumento baseado no método da gota pendente visualização da gota. Um controlador de temperatura com precisão de $\pm 0.5^{\circ} \mathrm{C}$ é usado para manter constante a temperatura do polímero até $300{ }^{\circ} \mathrm{C}$. O polímero é mantido no estado fundido em uma atmosfera de argônio para evitar degradação térmica.

O dispositivo de inserção consiste em uma seringa especialmente projetada para evitar problemas de capilaridade e de separação da gota (alongamento, pescoço) encontrados por outros pesquisadores ${ }^{9,33}$.

\section{Medições da tensão interfacial}

Usando o equipamento descrito pode-se obter a imagem de uma gota polimérica formada dentro de um segundo polímero. Os diferentes programas para cálculo da tensão interfacial a partir do perfil da gota são apresentados a seguir. Todo o processo é realizado em menos de um minuto.

O primeiro programa, após a digitalização da imagem, consiste em uma detecção de contorno (edge detection) da gota. O algorítmo é baseado em locação e extração de contornos binários do objeto (a gota). Após a detecção do contorno é necessário fazer uma correção desse contorno porque a resolução do digitalizador é limitada. O princípio do algorítmo foi descrito em detalhes em um trabalho anterior ${ }^{34}$. Uma vez corrigido, o perfil experimental da gota é comparado com a solução teórica da equação de Bashforth e Adams. Essa comparação de forma permite a avaliação da tensão interfacial. Detalhes sobre a comparação de forma podem ser obtidos em outros trabalhos já publicados ${ }^{35,36}$.

\section{Resultados}

O aparelho desenvolvido foi avaliado através de medidas da tensão interfacial entre n-hexano e água e entre n-octano e água. Esses dados são encontrados facilmente na literatura e portanto uma comparação entre os valores medidos com esse aparelho e os da literatura pode ser rapidamente realizada. A Tabela 1 apresenta os dados obtidos neste trabalho e os dados da literatura. As diferenças entre os dados obtidos neste trabalho e os da literatura podem ser atribuídas às diferentes temperaturas utilizadas.

Tabela 1. Tensão interfacial entre n-hexano e água e n-octano e água, utilizando o método da gota pendente

\begin{tabular}{cccc}
\hline Materiais & $\Delta \rho\left(\mathrm{g} / \mathrm{cm}^{3}\right)$ & $\begin{array}{c}\text { Valor determinado } \\
\text { neste trabalho } \\
(\text { dinas/cm) }\end{array}$ & $\begin{array}{c}\text { Valor da } \\
\text { literatura } \\
(\text { dinas } / \mathrm{cm})\end{array}$ \\
\hline n-hexano/água & 0,34 & 49,1 a $22^{\circ} \mathrm{C}$ & 51,1 a $20^{\circ} \mathrm{C}$ \\
n-octano/água & 0,31 & 48,4 a $22^{\circ} \mathrm{C}$ & 49,6 a $20^{\circ} \mathrm{C}$ \\
\hline
\end{tabular}




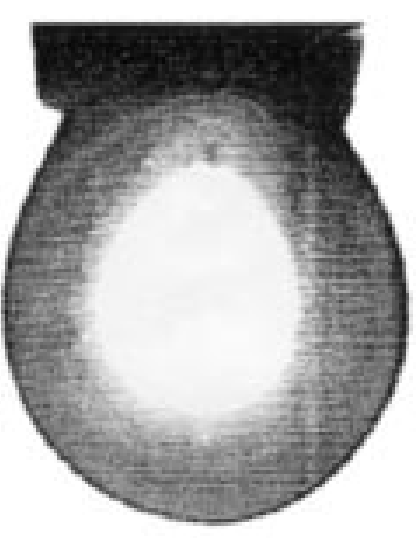

$3 a$

$3 b$
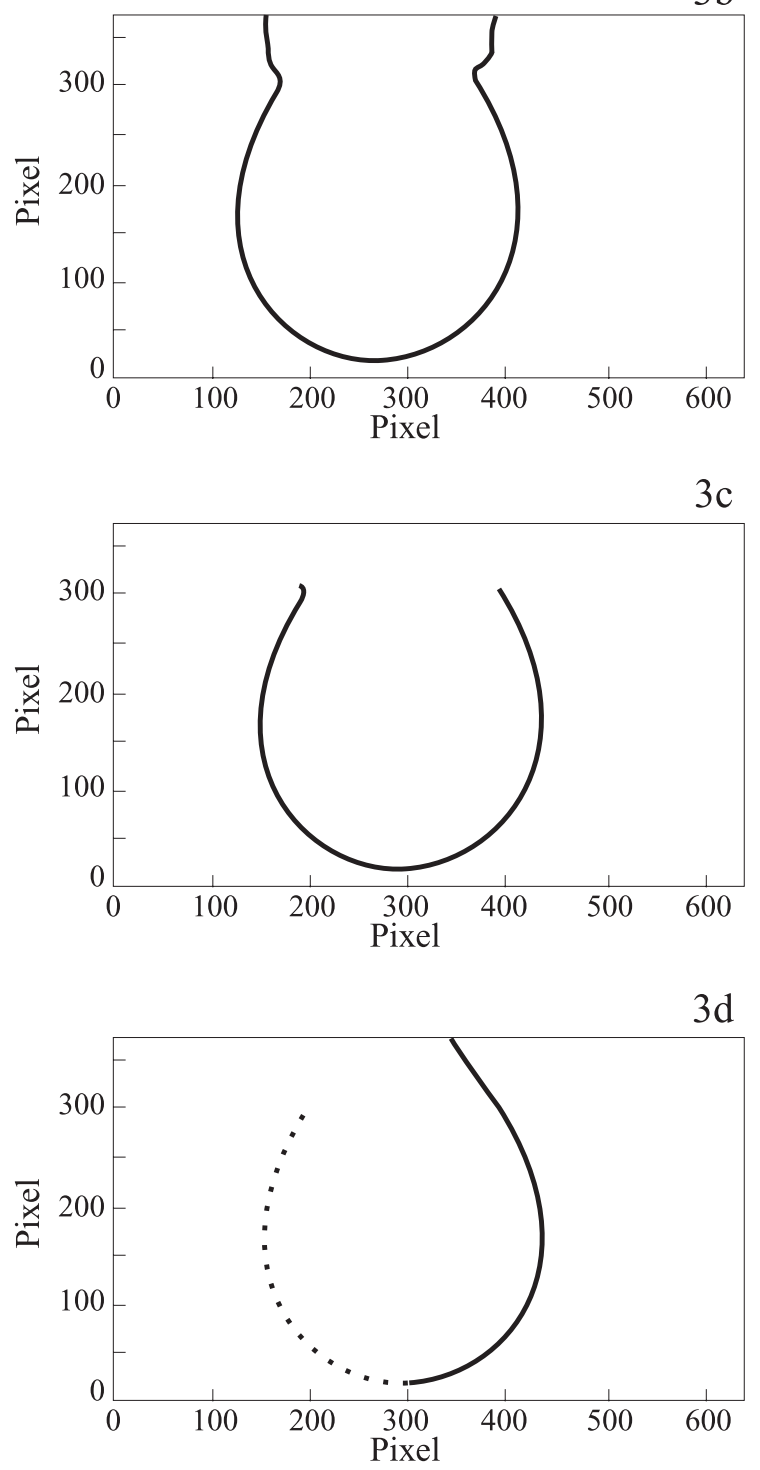

Figura 3. Gota de copolímero de etileno vinil álcool (EVOH) em polipropileno (PP) numa temperatura de $240^{\circ} \mathrm{C}$. 3a: Gota digitalizada; 3b: Gota depois da deteç̧ão de contorno; 3c: Gota depois da correção de contorno; 3d: Comparação de forma

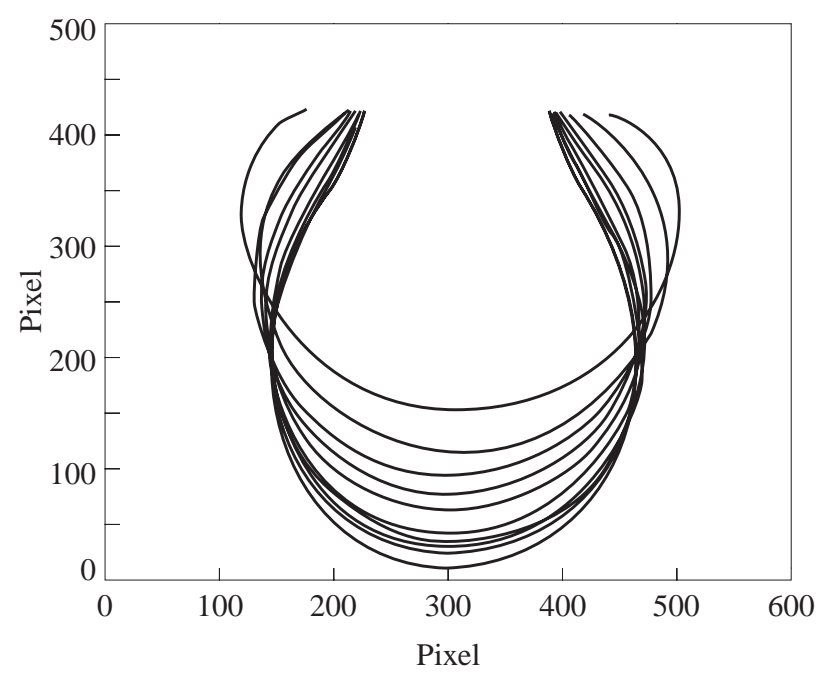

Figura 4. Evolução de uma gota de EVOH em PP em uma temperatura de $223^{\circ} \mathrm{C}$ em função do tempo

As figuras 3(a) a 3(d) mostram a seqüência de processamento de uma gota de um copolímero poli(etileno-co-álcool vinílico) $(\mathrm{EVOH})$ em polipropileno (PP) à temperatura de $240^{\circ} \mathrm{C}$ utilizando os programas descritos. A Figura 3(a) mostra a imagem digitalizada como pode ser vista na tela do computador. A Figura 3(b) mostra a gota depois da detecção de contorno. A Figura 3(c) mostra a gota depois da correção de contorno e a Figura 3(d) fornece a superposição da gota experimental (pontos) com o perfil teórico (linha) obtida usando a equação de Bashforth e Adams e comparação de formas. Devido à simetria, apenas metade da gota é mostrada. Deve ser mencionado que a escala das Figuras $3 b$ a $3 d$ é diferente da escala da Figura 3a.

A Figura 4 mostra a evolução, em função do tempo, de uma gota de EVOH em PP a temperatura de $223{ }^{\circ} \mathrm{C}$. Um contorno da gota é extraído a cada hora. O décimo contorno, o mais comprido, corresponde a nove horas de ensaios. Pode ser visto que o décimo primeiro contorno pode ser justaposto ao décimo. $\mathrm{O}$ equilíbrio mecânico foi portanto alcançado.

$\mathrm{O}$ aparelho da gota pendente foi usado para medir a tensão interfacial entre EVOH e PP para temperaturas variando de 210 até $250{ }^{\circ} \mathrm{C}$. Os polímeros são resinas comerciais com peso molecular alto. Também foi usado para avaliar a tensão interfacial para diferentes pares de polímeros como poliestireno/polipropileno, polietileno/poliestireno com pesos moleculares variando de 700 até $400.000 \mathrm{~g} /$ mol e temperaturas de 150 até $250{ }^{\circ} \mathrm{C}$. Os resultados obtidos podem ser encontrados em trabalhos já publicados $^{38,39}$. 
Método da gota girante "spinning drop"

\section{Princípio}

A deformação de uma gota girante de um liquido dentro de um outro líquido foi sugerida há muito anos como um possível método para se medir a tensão interfacial entre esses dois líquidos ${ }^{40}$.

O método da gota girante consiste em se introduzir uma gota de um liquido de densidade mais baixa num outro líquido de densidade mais alta em um tubo horizontal. O tubo gira em torno do seu eixo, em uma velocidade constante. Com o efeito da aceleração centrífuga, a gota sofre deformações, tomando a forma de uma elipse alongada (Figura 5). As dimensões finais da gota dependem essencialmente da velocidade do tubo, das diferenças de densidade entre as duas fases, e da tensão interfacial entre os dois líquidos. O conhecimento da velocidade do tubo e da diferença de densidade entre os dois líquidos permite a determinação da tensão interfacial.

Em velocidades elevadas, quando o comprimento da gota é maior do que quatro vezes o seu diâmetro, uma relação simples entre o diâmetro da gota, d, a diferença de densidade entre os dois líquidos, $\Delta \rho$, a velocidade angular do tubo, $\omega$, e a tensão interfacial, $\gamma$, pode ser escrita:

$$
\gamma=\frac{\Delta \rho \omega^{2} \mathrm{~d}^{3}}{32}
$$

Patterson e colaboradores ${ }^{14}$ foram os primeiros a utilizar o método da gota girante para determinar a tensão interfacial entre polímeros. Os autores determinaram a tensão interfacial entre poliisobutileno e poli(dimetil-siloxano). Verdier ${ }^{41}$ usou o método da gota girante para medir a tensão interfacial entre polietileno e poliestireno, poli(metacrilato de metila) (PMMA) e poliestireno, e PMMA e polietileno para temperaturas na faixa de $200^{\circ} \mathrm{C}$ até $250^{\circ} \mathrm{C}$.

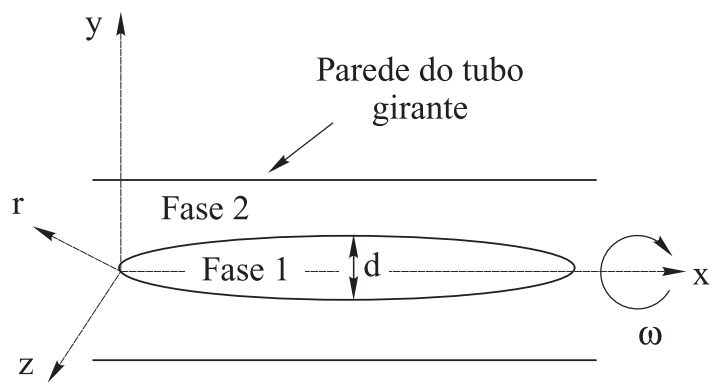

Figura 5. Geometria de uma gota girante
O método da gota girante é mais complicado do que os métodos da gota pendente ou séssil por causa do mecanismo rotativo. Entretanto, esse método envolve cálculos muito menos complexos e tempos muito menores para chegar ao equilíbrio mecânico do que o da gota pendente.

\section{Descrição do Instrumento}

O aparelho desenvolvido baseado no princípio da gota girante é constituido de três partes: uma câmara experimental montada sobre uma mesa provida de amortecedores de vibração, um sistema óptico para visualizar a gota e um sistema de aquisição de dados com um microcomputador para calcular a tensão interfacial do perfil da gota.

Um esquema da câmara experimental é mostrado na Figura 6. Ela consiste em um motor que gira o eixo onde é colocado o tubo de vidro contendo o polímero, um forno e uma sonda de capacitância, para medir a expansão volumétrica do polímero. $\mathrm{O}$ motor tem uma velocidade máxima de rotação de 40.000 rpm. Os mancais especiais usados para a rotação do tubo estão localizados sobre aletas de refrigeração. O forno é constituído de duas partes, sendo que a parte

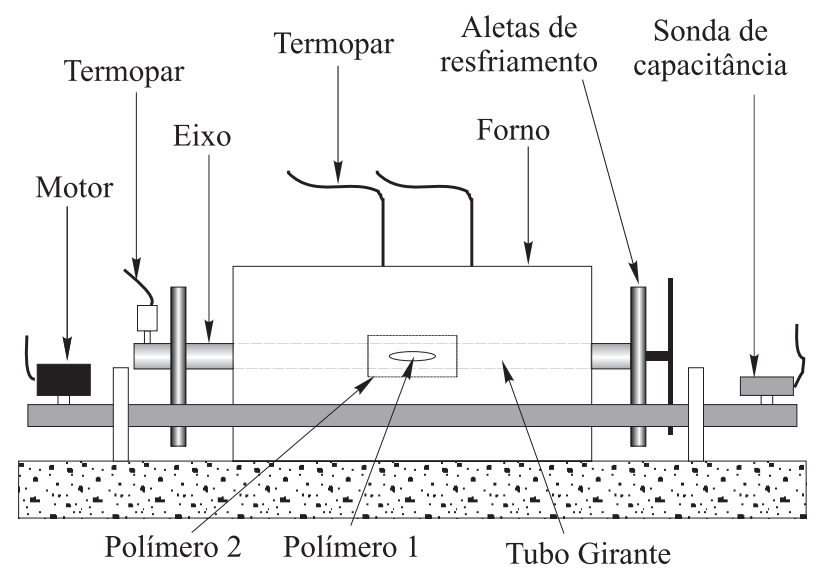

Figura 6. Esquema da montagem do instrumento baseado no princípio da gota girante

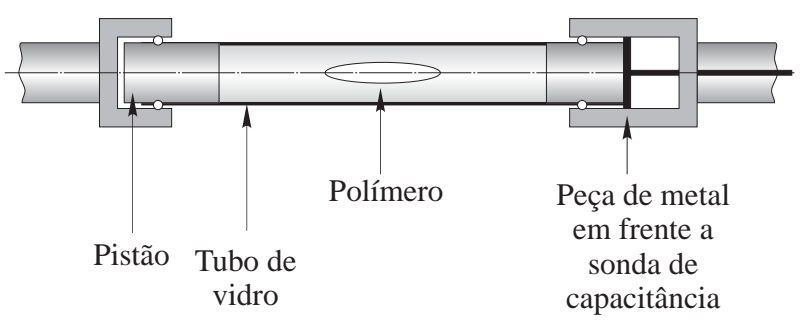

Figura 7. Tubo de vidro do intrumento baseado no princípio da gota girante 
superior pode ser removida facilmente. Uma cavidade no centro do forno permite a rotação do tubo. $\mathrm{O}$ forno contém duas janelas, uma de cada lado do forno, para permitir a observação e a iluminação dos polímeros. A temperatura no centro do forno é medida com o auxílio de um termopar. O forno pode ser aquecido até $400{ }^{\circ} \mathrm{C}$. A temperatura é controlada com um controlador de temperatura P.I.D. (Proportional Integrator Derivator) com precisão de $\pm 0.5^{\circ} \mathrm{C}$. O tubo de vidro é feito de boro silicato. O diâmetro externo é de $1 / 2$ polegada e o diâmetro interno é de $3 / 8$ de polegada. O tubo de vidro é fechado usando um pistão de vidro em cada um dos lados. Um dos pistões de vidro é móvel para ajustar a expansão do polímero. A Figura 7 mostra o tubo de vidro.

O sistema óptico para iluminar e visualizar a gota é o mesmo que no caso da gota pendente. Somente o jogo de lentes é diferente por causa da diferença de tamanho da gota.

\section{Medidas de Tensão Interfacial}

Usando o equipamento descrito pode-se obter a imagem de uma gota polimérica formada dentro de um segundo polímero. Como no caso do aparelho baseado no princípio da gota pendente, a imagem da gota é digitalizada no "frame-grabber" instalado no microcomputador. As dimensões da gota podem ser obtidas da imagem digitalizada. Conhecendo-se a diferença de densidade entre os dois polímeros e a velocidade rotacional é possível calcular a tensão interfacial usando a equação (2).

\section{Resultados}

As Figuras 8(a) até 8(e) mostram as imagens digitalizadas de uma gota de polipropileno (PP) no copolímero de poli(etileno-co-álcool vinílico) a $202{ }^{\circ} \mathrm{C}$. As figuras mostram a evolução no tempo de uma gota tendo como velocidade de rotação 16.000 rpm no a) começo do ensaio, b) após 12 minutos, c) após 110 minutos, d) após 210 minutos e e) após 300 minutos. Após 150 minutos a forma da gota não variou mais com o tempo como pode ser visto nas Figura 8(d) e 8(e).

O aparelho foi utilizado para determinar a tensão interfacial entre polipropileno e o copolímero poli(etileno-co-álcool vinílico) para temperaturas variando entre $202{ }^{\circ} \mathrm{Ce} 224^{\circ} \mathrm{C}$. Os resultados podem ser encontrados em um artigo anteriormente publi-

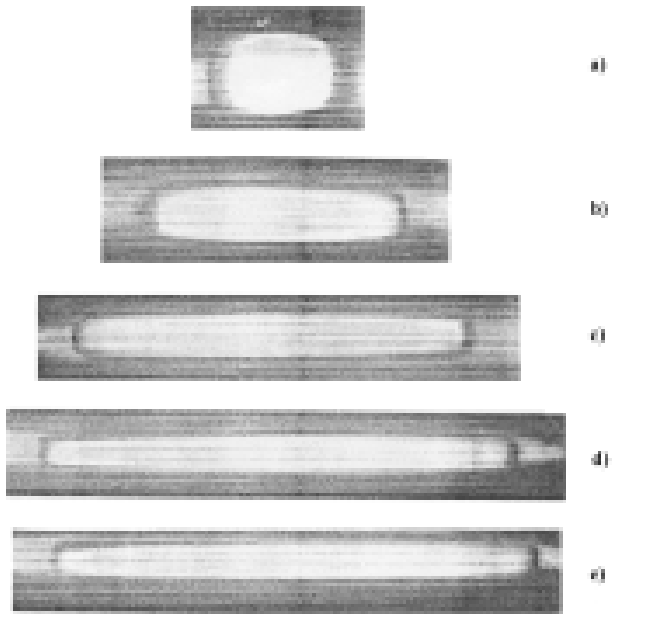

Figura 8. Evolução de uma gota de PP em EVOH em uma temperatura de $202{ }^{\circ} \mathrm{C}$

cado $^{34}$. O aparelho foi utilizado também para determinar a tensão interfacial entre polipropileno maleatado (MAgPP), (polipropileno modificado) e copolímero poli(etileno-co-álcool vinílico) (EVOH). Não foi possível medir a tensão interfacial entre esses dois polímeros com um aparelho baseado no método da gota pendente. A degradação ocorreu antes de se chegar ao equilíbrio mecânico requerido para medições da tensão interfacial. Também, o polipropileno maleatado, o polímero de densidade mais baixa no par MAgPP/EVOH é opaco. No caso de medições com o aparelho baseado no princípio da gota pendente, o polímero com densidade mais baixa forma a matriz e portanto deve ser transparente afim de permitir a observação da evolução da gota. No caso de medições com o aparelho baseado no método da gota girante o material de densidade mais baixa forma a gota e portanto medições foram possíveis no caso do par MagPP/EVOH.

\section{Comparação entre os dois métodos}

O método da gota pendente tem as seguintes vantagens quando comparado com o método da gota girante para medir a tensão interfacial entre polímeros:

a) A solução de Bashforth e Adams usada no método da gota pendente precisa de um número menor de suposições do que a equação de equilíbrio usada no caso da gota girante.

b) Quando o material de densidade maior é opaco, não é possível utilizar o método da gota girante.

c) Para fazer uma determinação de tensão interfacial necessita-se de menor quantidade de polímero. 
As desvantagens do método da gota pendente quando comparado com o método da gota girante são as seguintes:

a) Os tempos para chegar ao equilíbrio são maiores. Isso pode impedir algumas medições devido à ocorrência de degradação térmica.

b) Quando o material de densidade mais baixa é opaco, não é possível se usar o método da gota pendente.

c) Problemas de capilaridade ou destacamento da gota podem ser encontrados.

\section{Conclusão}

Dois aparelhos, um baseado no princípio da gota pendente e um outro baseado no método da gota girante foram descritos. Com ambos aparelhos foi possível determinar-se a tensão interfacial entre polímeros "on-line". Os dois aparelhos são complementares quanto aos polímeros estudados. As vantagens e desvantagens de cada um dos aparelhos foram apresentadas.

\section{Agradecimentos}

Os autores agradecem à FAPESP (Proc. 94/3351-6)

\section{Bibliografia}

1. Wu S.: Polymer Eng. Sci., Vol 27(5), p 335, (1987)

2. Van Oene : J. Colloid. Inter. Sci., Vol 40, p 448 (1972)

3. Xanthos M., Young M.W. and Biesenberger J.A.: Polym. Eng. Sci. Vol 30(6),p 355 (1990).

4. Wu S. : J. Colloid Interface Sci., Vol 31, p 153 (1969).

5. Roe R.J. : J. Colloid Interface Sci., Vol31, p 228 (1969).

6. Wu S.: Polymer Interface and Adhesion, Marcel Dekker, Inc., NY, (1982).

7. Anastasiadis S.H.: "Interfacial Tension of Immiscible Polymer Blends", Ph.D. Thesis, Princeton University, (1988).
8. Demarquette N.R., Kamal M.R.,: Antec's Proceedings, p 1907-1911, Detroit (1992)

9. Demarquette N.R. and Kamal M.R. : Pol. Eng. Sci., Vol 34, No24, p 1823-1833 (1994).

10. Kamal M.R., Demarquette N.R., Lai-Fook R.A., Price T.A., "Evaluation of thermodynamic theories to predict interfacial tension between polystyrene and polypropylene melts" vol 37, p 813-825 (1997).

11. Staicopulus D.N.: J. Interface Sci., Vol 17, p 439 (1962).

12. Staicopulus D.N.: J. Interface Sci., Vol 18, p 793 (1963).

13. Staicopulus D.N.: J. Interface Sci., Vol 23, p 453 (1967).

14. Patterson H.T., Hu K.H. and Grindstaff T.H.: J. Polym. Sci., Part C, Vol 34, p 31 (1971).

15. Elmendorp J.J. and de Vos G.:Polym. Eng Sci., Vol 26, p 415 (1986).

16. Joseph D.D., Arney M.S., Gillberg G., Hu H., Hultman D., Verdier C., Vinagre T.M.: J. Rheol., Vol 36(4), p 623 (1992).

17. Demarquette N.R., Kamal M.R., R.A . Lai Fook, H. Garmabi: "A spinning drop apparatus to measure interfacial tension between polymers", sendo revisto.

18. Elemans P. and Janssen J. : J. Rheol, Vol 34, p 781 (1990).

19. Carriere C.J., Cohen A. and Arends C.B.: J. of Rheol., Vol 33 (5), p 681 (1989).

20. Jon D.I.: "Measurements of low and ultralow interfacial tension by means of the blade, spinning drop, pendant drop and laser light scattering method", Ph.D Thesis, University of New York, (1986).

21. Sauer B.B., Skarlupka R.J., Sano M. and Yu H: Polymer Preprints, Vol 28, p 20 (1987).

22. Grassmespacher H. and Meissner J.: J. Rheol., Vol 36(6), p 1127 (1992).

23. Graebling D. and Muller R.: Colloids and Surfaces, Vol 55, p 89 (1991).

24. Schonhorn H., Ryan F.W., and Sharpe L.H.: J. Polym. Sci., A-2, Vol 4, p 538 (1966). 
25. Edwards H.: J. Appl. Polym. Sci., Vol 12, p 2213 (1968).

26. Hartford J.R.J., and White E.F.T.: Trans. J. Plastics Inst., Vol 53 (1969).

27. Dettre R.H. and Johnson R.E. Jr.: J. Colloid Interface Sci., Vol 21, p 367 (1966).

28. Du Nouy L. : J. Gen. Physiol., Vol 1, p 521 (1919).

29. Newman S.B. and Lee W.L. : J.Polym Sci., Part B, Vol 3, p 235 (1958).

30. Schonhorn H. and Sharpe L.H.: J. Polym. Sci., Part B, Vol 3, p 235 (1965).

31. Padday J.F. : in Surface and Colloid Science, Vol 1, E. Matijevic Ed., John wiley \& Sons, New York, (1969).

32. Bashforth S. and Addams J.C.: An attempt to test the Theory of Capillary Action, Cambridge University Press and Deighton, Bell and Co, London (1882).

33. Escudie E.: Material Chemistry and Physics, Vol 14, p 239 (1986).
34. Demarquette N.R. : "Interfacial Tension in Polymer Blends: Measurements and Analysis", Ph.D. thesis, McGill University, Montreal, Canada (1993)

35. Siegel A . F.: Biometrica, Vol 69, p 242 (1982)

36. Siegel A . F., Benson R.H.: Biometrica, Vol 38, p 341 (1982)

37. Perry R.H. and Chilton, H.: "Chemical Engineers Handbook", McGraw Hill, New York, (1973)

38. Kamal M.R., Lai Fook R.A. e Demarquette N.R., Polym. Eng. Sci., Vol 34, N 24, p 1834-1839 (1994)

39. N.R. Demarquette, M.R. Kamal, R.A . Lai Fook, Ano VI, Revista da ABPol, Revista Polímeros Ciência e Tecnologia, Ano VI, pp 38-43, No3Jul/Set (1996)

40. Vonnegut B. : Rev. Sci. Instrum., Vol 13, p 6 (1942).

41. Verdier C. : Topics in the fluid mechanics of viscoelastic liquids, Ph.D. Thesis, University of Minesota, (1990). 